http://jmscr.igmpublication.org/home/ ISSN (e)-2347-176x ISSN (p) 2455-0450

crossref DOI: https://dx.doi.org/10.18535/jmscr/v7i9.64

Journal Of Medical Science And Clinical Research

IGM Publication

An Official Publication of IGM Publication

\title{
Improving Early Diagnosis of Cervical Cancer Lesions Using P16 INK4a Biomarkers on Cellblocks from Cervical Smears
}

\author{
Authors \\ Martha W. $M^{1^{*}}$, Kyama M.C ${ }^{2}$, Kibet P.S ${ }^{3}$
}

${ }^{1}$ Jomo Kenyatta University of Agriculture and Technology, Department of Medical Laboratory Sciences

${ }^{2}$ University of Nairobi Department of Pathology

${ }^{3}$ Kenyatta National Hospital

*Corresponding Author

Martha W. Maina

Jomo Kenyatta University of Agriculture and Technology, Department of Medical Laboratory Sciences

\section{Abstract}

Universally, cancer of cervical is the second utmost regular malignant growth in females after cancer of breast. Timely recognition of pre-malignant lesions is an essential segment for the decrease of related morbidity and mortality. Though Pap test has been a helpful screening device, however hampered by high Intra and inter-observer changeability, false negatives at 20-30\% and false positives at 5-70\%.

Objective: The study determined the role of P16 INK4a biomarker in the identification of low grade squamous intra-epithelial abrasions in cell blocks prepared from Pap smears and subsequently compared previous Pap results with colposcopy and cellblock results

Methods: This was a laboratory-based, prospective study with a parallel comparative arm at the Kenyatta National Hospital Reproductive health clinic (66). All patients who had abnormal Pap smear reports and referred for colposcopy, and consented for the study were enrolled. A smear was taken just before taking a colposcopy biopsy. The cytobrush was immediately put in Acid alcohol fixative centrifuged and deposits wrapped in a filter paper and processed histologically to form a cellblock. Colposcopy biopsies were then retrieved from the KNH histology lab and both samples subjected to Routine histological stain and eventually with biomarker P16. Total of 85 samples was collected.

Results: There was a significant level of agreement between Pap smears and cellblock findings on the routine Histological stain. Of 58 cases analyzed Colposcopy had (39\%) 27 negatives and (45\%) 31 positives while cellblock had (48\%) 33 negative and (36\%) 25 positive for pre and malignancy with a confidence interval of 0.016 as the margin of error. Biomarker Colposcopy had (43\%) 30 negativity and (41\%) 28 positivity while cellblock had negativity of (46\%) 32 and positivity of (38\%) 26. The specificity and sensitivity were based on the true values of colposcopy as the golden standard test for cervical cancer. Biomarker cellblock had the "sensitivity and specificity" of $93.3 \%$ and $93.8 \%$ respectively, while Haematoxyline and eosin-stained cellblock had sensitivity and specificity of $83.8 \%$ and $81.8 \%$ respectively. Conclusion: Poor inter-rater agreement resulting to mortality and morbidity associated with false positives and false negatives, cellblock prepared from residues of cytobrush stained with haematoxyline and eosin and biomarker is likely to circumvent all the above, together with minimizing loss to follow up as patients only visit health facility once and they acquire all the results without re-sampling hence drastically reducing the cost of colposcopy, which requires highly specialized equipment and experienced personnel who are very few and difficult to find. 


\section{Introduction}

Globally, cervical malignancy is the second most regular disease in ladies after breast cancer ${ }^{[1]}$. An opportune location of pre-malignant sores is a critical part to decrease the related morbidity and mortality ${ }^{[2]}$. Pap test has been a helpful "screening tool however hampered by high inter and intraobserver fluctuation" "[3]. This has brought about "high false negative and false positive rates that extend between $20-30 \%$ and 5- $70 \%$

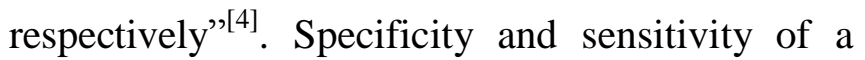
Pap smear for HSIL using LBC have not been shown to improve though ${ }^{[5]}$. Colposcopic cervical biopsies are "considered as the gold standard, though hampered by intra- and inter-observer variability" $^{,[6]}$.

HPV testing is productively incorporated into the essential screening, either as an assistant to cytology or as a sole essential test. In any case, "it fails in the triage of low-grade lesions", and regardless of whether actualized as an essential screening test, it is "important to have a more disease explicit triage marker to distinguish women that would need to undergo colposcopy"[7]. Moreover, a solitary DNA test of Human Papilloma Virus affirms "infection by the virus, present in every cervical malignancy" at 99.7\%; it doesn't segregate among transient and unending disease ${ }^{[8]}$. Performing IHC on cervical smears could evade this impediment ${ }^{[9]}$, circumvent invasive interventions and avert possibly increased morbidities by using target choices at the negligibly intrusive cervical cytology stage.

The epidemiology of cervical cancer, just as experimental examinations, demonstrates that the development of cervical cancer predetermined by various etiological factors, but the "infection with the human papilloma virus (HPV) is the utmost factor towards the development of disease"[10]

In squamous cell carcinomas, HPV DNA is available in about $95 \%$, while in adenocarcinoma of the cervix, has been recognized in just a range of $70 \%$ to $90 \%^{[10]}$. In most developed and some developing nations, the frequency of squamous cell carcinoma has reduced remarkably because of population-based Pap smear screening. "There has been a relative increment in the rate of adenocarcinoma among women" amid a similar period $^{[7]}$. A few investigations have detailed this escalation, particularly in young women.

The development of adenocarcinomas cannot be effectively prevented by cytological screening alone ${ }^{[11]}$. The various grades of dysplasia can be used to distinguish the precancerous phases of invasive cervical carcinoma and characterize the stages of development. It is proposed that around " $12 \%$ of all carcinomas in-situ, when left untreated, advanced into obtrusive malignancy after 13 years" ${ }^{,[12]}$. "History of cervical intraepithelial neoplasia (CIN) is extremely variable and unpredictable when left untreated, CIN may revert to normal", either persist or eventually advance to intrusive cervical malignant growth. The investigation of those that will progress or relapse has critical clinical value ${ }^{[13]}$

P16INK4a, a cancer silencer genetic factor, is a "component of the P16INK4a/cyclinD-cdk4/6/Rb pathway" ${ }^{\text {"14] }}$. It can initiate G1 cell cycle capture by repressing the "phosphorylation of $\mathrm{pRb}$ by $\mathrm{cdk} 4$ and cdk6",[12]

Current investigations demonstrates that immunohistochemical stain techniques with "P16INK4a is a promising marker for dysplastic and cancerous cervical epithelia" ${ }^{,[15]}$. Different examinations have likewise shown that over-expression of P16INK4a aids the recognition of "high-hazard HPV-related cervical squamous lesion" "[16]. It was suggested that $\mathrm{P} 16^{\mathrm{INK} 4 \mathrm{a}}$ is valuable for recognizing immature squamous metaplasia which forms a "high-grade squamous intra-epithelial lesion", where the previous could act like a morphologic discrepancy finding ${ }^{[17]}$.

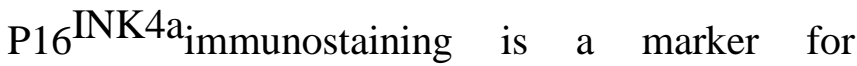
identifying dysplastic lesions caused by HR HPV and in distinguishing non-HPV-related changes. Some of the changes are such as squamous metaplasia, inflammatory conditions or reactive conditions either to mask or mimic dysplasia in the cervical biopsy specimen and the HPV- related 
changes like CIN. "P16 ${ }^{\mathrm{INK} 4 \mathrm{a}}$ has recently been described as a surrogate marker for HR-HPV and in glandular intraepithelial lesions of the cervix" and associated squamous ${ }^{[18]}$. It also demonstrates that the immuno-histochemical staining pattern of P16 ${ }^{\text {INK4a }}$ is different in high-grade intraepithelial neoplasia of the uterus (CIN 2 and 3), where it is diffuse, from sporadic or focal staining patterns in CIN $1^{[19]}$.

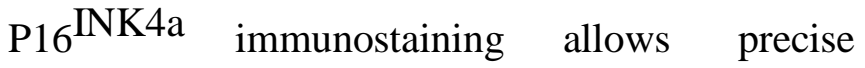
identification of even small cervical cancer lesions or CIN in sections of biopsy and helps in variation reduction of inter-observer in interpretation of histopathologic cervical biopsy specimens $^{[22]}$. Thus, false-negative and falsepositive biopsy interpretation can reduce by p16 IHC and thereby improve (pre)-cancer diagnosis of the cervix significantly. Also, $\mathrm{P} 16^{\mathrm{INK} 4 \mathrm{a}}$ negative CIN1 may benefit from a less intensive follow-up as they rarely progress to high grade.

The screening tests currently existing $\mathrm{have}$ significant disadvantages dur to their inability to differentiate "lesions that will progress and those that do not" ${ }^{\text {"20] }}$.To surmount limitations; the tests existing currently requires an indication "that an oncogenic HPV virus has rendered infected cells susceptible to transformation" through enhanced genetic instability leading to the development of cancer. The virus exerts its oncogenic potential in a particular woman as the test for HR HPV indicates ${ }^{[12]}$

A number of studies have focused on the utility of

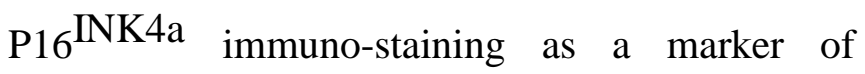
identifying dysplastic lesions caused by HR HPV and also in distinguishing non-HPV-related changes, such as squamous metaplasia, inflammatory conditions or reactive conditions that either mask or mimic dysplasia in cervical biopsy specimen; from HPV-related changes like CIN 1 $\left({ }^{[21]}\right.$. It has been also demonstrated that the pattern of $\mathrm{P}^{\mathrm{INK}} \mathrm{I}^{\mathrm{IN}}$ immunohistochemical staining is different in "high-grade intraepithelial neoplasia of the cervix (CIN 2 and 3)", where it is diffuse, from sporadic or focal staining patterns in CIN $1^{[22]}$

According to Klaes et al., the CIN 2/CIN 3, CIN1 associated with high-risk human papillomavirus, or cervical cancer restricted the expression of P16 ${ }^{\text {INK4a }}$ Which now has a significant better agreement in the interpretation. Thus, falsenegative and false-positive biopsy interpretation can reduced by p16 IHC and improve cervical (pre)-cancer diagnosis significantly. $\mathrm{P} 16^{\mathrm{INK} 4 \mathrm{a}}$ negative CIN1may benefit from a less intensive following up as they rarely progress to high grade [19].

\section{Aim}

To determine the role of $\mathrm{P} 16^{\mathrm{INK} 4 \mathrm{a}}$ in early diagnosis of cervical cancer from cervical lesions using cellblocks in patients referred for colposcopic biopsy.

\section{Methodology \\ Study Site}

The study was carried out at $\mathrm{KNH}$ 's reproductive health unit clinic (clinic 66). The special clinic handles gynecological related complications.

\section{Study Design}

This was a laboratory-based, prospective study with a parallel comparative arm.

\section{Data Collection Methods}

Patients whose Pap smear reports had been recommended for colposcopy were recruited for the study. Those who gave consent had smear samples taken using a cytobrush. The cytobrush was put in a labeled centrifuge tube containing AA fixative and cocked ready for transportation to the laboratory. They were then centrifuged, supernatant discarded and the brush discarded. $10 \mathrm{ml}$ of the fixative was added to each of the deposits centrifuged and the supernatant discarded. This procedure was repeated 2 times. $10 \%$ formalin was added and left to stand for $4 \mathrm{hrs}$. The deposits were picked using forceps wrapped in a filter paper and processed in a tissue processor, embedded and cellblocks prepared.

The corresponding colposcopic biopsies were 


\section{JMSCR Vol||07||Issue||09||Page 362-369||September}

retrieved from a Histopathology laboratory at Kenyatta National Hospital and both the cellblocks and Colposcopy biopsies were subjected to routine histological tests ( $\mathrm{H} \& \mathrm{E})$ and the P16 biomarker tests. The PI interpreted results together with the pathologist and recorded the outcomes.

\section{Results}

69 (N) samples were collected from a study population for analysis out of which a sample population (n) of 58 met the inclusion criteria. The sampling procedure was based on the previous Pap smear test results were in this study, Pap smears had either HSIL, LSIL, ASCUS AGUS

Table 1 The overall distribution of the tests

\begin{tabular}{|l|c|c|}
\hline & Negative & Positive \\
\hline Biomarker cellblock & 32 & 26 \\
\hline Biomarker colposcopy & 30 & 28 \\
\hline HE cellblock & 33 & 25 \\
\hline HE colposcopy & 27 & 31 \\
\hline Pap smear & 26 & 32 \\
\hline
\end{tabular}

From Table 1 above,
58 of 69 cases analyzed biomarker Colposcopy had (43\%) 30 negativity and (41\%) 28 positivity while cellblock had negativity of (46\%) 32 and positivity of (38\%) 26

Colposcopy had (39\%) 27 negatives and (45\%) 31 positives while cellblock had (48\%) 33 negative and $(36 \%) 25$ positive for pre and malignancy.

Table 2

\begin{tabular}{|lr|ll|}
\hline \multicolumn{2}{|c|}{ CELLBLOCK } & \multicolumn{2}{c|}{ COLPOSCOPY } \\
\hline \multicolumn{2}{|c|}{ POSITIVE } & \multicolumn{2}{c|}{ POSITIVE } \\
\hline LSIL & 11 & CIN1 & 16 \\
\hline HSIL & 9 & CIN2 & 10 \\
\hline ASCUS & 5 & CIN3 & 5 \\
\hline TOTAL & 25 & TOTAL & 31 \\
\hline
\end{tabular}

Of 58 cases on the routine histological stain (haematoxyline and eosine) carried out, LSIL were $11(18 \%)$ of all cases HSIL $9(15 \%)$ ASCUS $5(8 \%)$ on cellblock and on colposcopy CIN1 were $16(28 \%)$ cases CIN2 $10(17 \%)$, CIN3 5 $(8 \%)$.

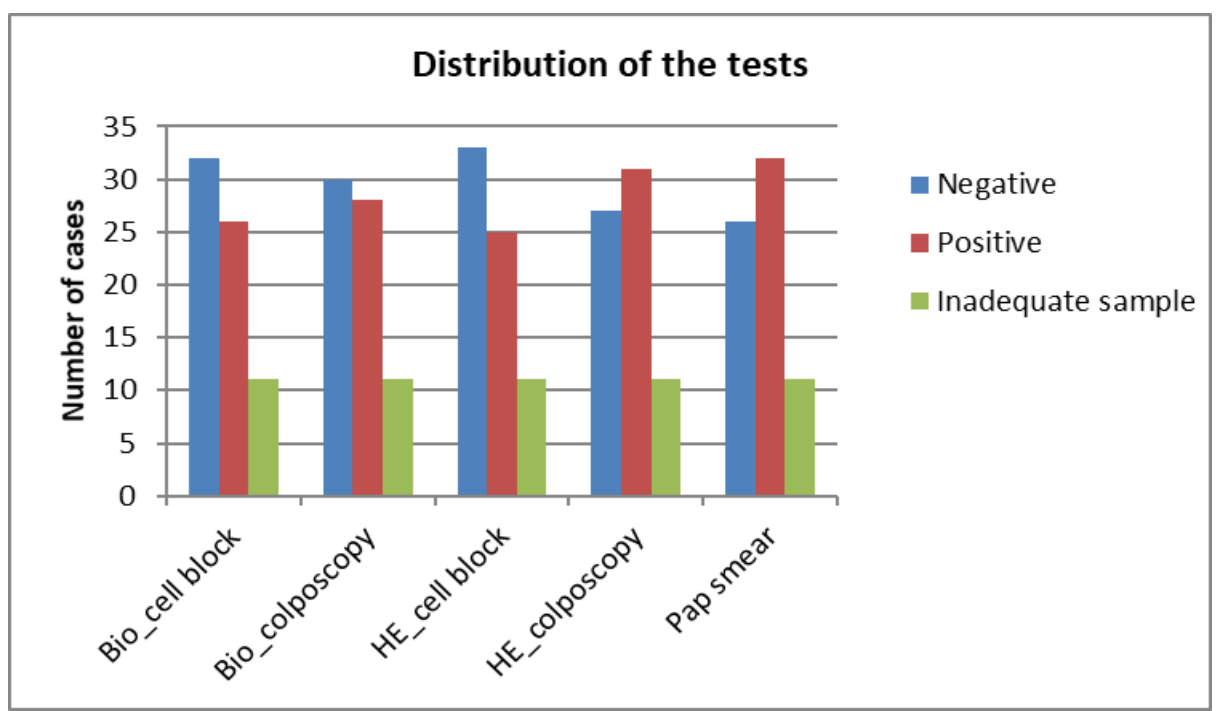

The table for $\mathrm{P}$ values

Table 1: Biomarker cellblock and colposcopy and pap

\begin{tabular}{llllll}
\hline Biomarker Cellblock & & Negative & Positive & Total & P-Values \\
\hline Biomarker Colposcopy & Negative & 21 & 9 & 30 & \\
& & & & & 0.018 \\
& Positive & 11 & 17 & 28 & \\
\cline { 2 - 6 } Total & 32 & 26 & 58 & \\
\cline { 2 - 6 } Pap Smear & & & & & \\
& Negative & 18 & 8 & 26 & 0.046 \\
& Positive & 14 & 18 & 32 & \\
\hline & Total & 32 & 26 & 58 & \\
\hline
\end{tabular}


Table 2: H\&E cellblock colpo \&

Pap smear

\begin{tabular}{llcccc}
\hline Haematoxyline \& eosine Cellblock & & & & & \multirow{2}{*}{ P-values } \\
\hline Haematoxyline \& eosine Colpo & Negative & 19 & 8 & 27 & \\
& Positive & 14 & 17 & 31 & 0.047 \\
& Total & 33 & 25 & 58 & \\
Pap Smear & Negative & 21 & 5 & 26 & \multirow{2}{*}{0.001} \\
& Positive & 12 & 20 & 32 & \\
& Total & 33 & 25 & 58 & \\
& & & & & \\
& & & &
\end{tabular}

\section{Specificity and sensitivity of the tests}

\begin{tabular}{|l|c|}
\hline Test Results & Reality \\
\hline Biomarker Cellblock & Biomarker Colposcopy \\
\hline H\&E Cellblock & H\&E Colposcopy \\
\hline
\end{tabular}

Table 3: Biomarker cellblock specificity and sensitivity calculations using Biomarker Colposcopy as the golden standard for the true positive and true negative values.

\begin{tabular}{lcc}
\hline & Sensitivity & specificity \\
\hline Biomarker cellblock & $93.3 \%$ & $93.8 \%$ \\
\hline
\end{tabular}

Table 4: H\&E cellblock specificity and sensitivity calculations using H\&E colposcopy as the golden standard for the true positive and true negative values.

\begin{tabular}{lcc}
\hline & sensitivity & specificity \\
\hline H\&E Cellblock & $83.8 \%$ & $81.8 \%$
\end{tabular}

From the table 2: it was observed that there was significance in the Biomarker tests carried on the cellblock and colposcopic biopsies having a significance of 0.018 which is below 0.05 indicating a level of agreement and hence a cellblock could be used in place of the colposcopic biopsy.

Similarly, for the routine histological stain, there was significance in both the cell block and colposcopic biopsy, Cellblock and Pap smear with $\mathrm{P}$ values of 0.047 and 0.001 respectively indicating that cell block remains a useful screening and diagnostic tool for early cervical cancer detection.

\section{Expression of P16ink4a on Cell Blocks \& Colposcopy Biopsies}

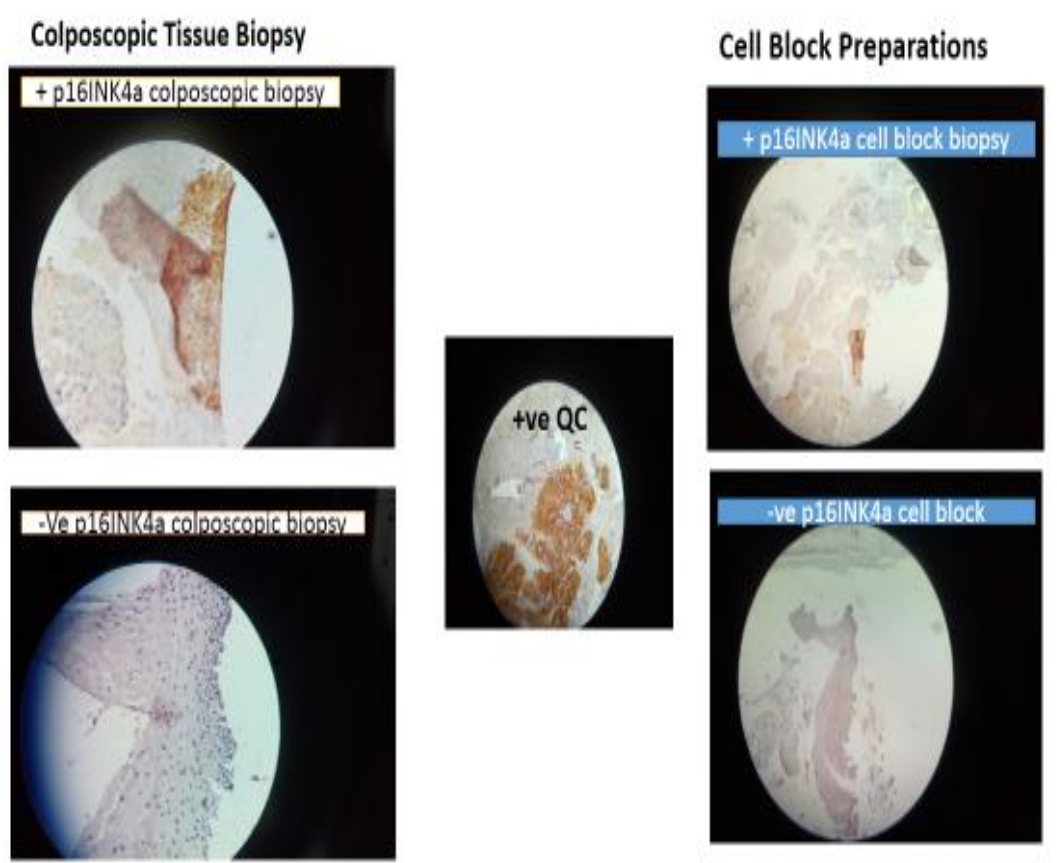




\section{Discussion}

P 16 is a widely used surrogate marker for HPV related cervical dysplasia. It is believed to have a high diagnostic efficacy in the determination of a transient and chronic HPV infection.

The study enrolled 69 participants whose samples were collected out of which 58 met the inclusion criteria, and 11 samples were inadequate to proceed for investigation. The age of the participants ranged from 21 to 70 years with a mean age of 43.2029 and the standard deviation of 9.771778. The sampled age range comprised of three divorced females, 52 married and 14 singles. There was a significant level of agreement between Pap smears and cellblock findings on the routine Histological stain. Of 58 cases analyzed Colposcopy had (39\%) 27 negatives and (45\%) 31 positives while cellblock had (48\%) 33 negative and (36\%) 25 positive. Biomarker Colposcopy had (43\%) 30 negativity and (41\%) 28 positivity while cellblock had negativity of $(46 \%) 32$ and positivity of (38\%) 26.

Pap smear compared to biomarker cellblock had a p-value of 0.046 showing a significance agreement and the routine histology stain for cellblock and Pap smear had a p-value of 0.001. This indicated that a cellblock can be useful for screening and diagnosis as there was a significant level of agreement between the two tests. For this reason, there will be a minimal loss to follow up and hence avoid the need for highly specialized and significantly cut the cost of screening equipment currently in use for the gold standard.

There was a considerable variation on the number of negatives and positives between colposcopy and cellblock. The difference in the variation of cellblock on H\&E and Biomarker could be as a result of cellblock preparations sampling techniques, processing of colposcopic biopsies, the degradation of the tissue block and the existing inter and intra-observer variability.

The sensitivity was based on colposcopy as a golden standard taking its results as true values for both negative and positive results. Colposcopy is regarded as a gold test/confirmatory test for cervical cancers $^{[40]}$. Biomarker cellblock showed a sensitivity at $93.3 \%$ while the H\&E cellblock demonstrated the sensitivity of $83.8 \%$. The theoretical sensitivity of cellblock ranges from $90 \%$ to $94.5 \%$, but the sensitivity can be increased for cyto- diagnosis at analytical stages. The theoretical sensitivity of colposcopy known to range from $87 \%$ to $99 \%$.

Specificity was based on the true values of colposcopy. The experimental specificity values for $\mathrm{H} \& \mathrm{E}$ cellblock is $81.8 \%$ and the specificity of Biomarker cellblock $93.8 \%$ as compared to the theoretical specificity of colposcopy of up-to $98.5 \%$.

For cervical screening, p16 is still an excellent surrogate marker highly useful for detection of dysplastic lesions. The cell cycle regulator over expressed in squamous dysplasia and cellblock together with colposcopy can be used to further diagnostic accuracy of dysplastic lesions, mitigate inter and intra-observer variation, loss to follow up, late presentation of patients, unnecessary invasive procedures and misdiagnosis resulting to mismanagement.

The gold standard test for cervical malignancy, stained with $H \& E$ gives a false impression of a static process that cannot demonstrate the progression from pre-to malignancy. Compared to a cellblock with $\mathrm{H} \& \mathrm{E}$ and $\mathrm{BM}$ having the potential to distinguish CIN from other lesions and distinguish chronic from the transient.

Cellblock if well prepared and well sampled could serve as a tool for screening, diagnosing and a confirmatory for pre and malignant cervical lesions as it provides multiple sections paving way for additional markers that may be used for further testing and use of other diagnostic markers that would improve on patient's management.

The p16 expression has been associated with glandular intraepithelial lesions and neoplastic squamous. p16 over expression resulting from a futile feedback loop, generated in "complex molecular mechanisms where high-risk HPV transforming proteins interact with cell cycle proteins" ${ }^{[39]}$. Diffuse expression "represents a 
useful means for the pathologist to separate lesions requiring colposcopy from those that do not". Through immuno-peroxidase study, in the transformed high-risk HPV-related cervical lesions, it is the expression of p16 that serves as a surrogate marker ${ }^{[40]}$.

"The failure to provide abnormal tissue of sufficient amount and depth is the single most common cause of an inadequate biopsy specimen" [41]. Invasive neoplasm is likely to be interpreted as an in-situ lesions if it is without the underlying stroma. On the prognostic significance of p16 especially in LSIL prediction of regression or progression to malignancy.

\section{Conclusion}

The two histological testing techniques; colposcopy and cellblock, agree on the variables examined. There was a considerable variation on the number of negative and positive results between the histological assessment using the Pap test as compared to both colposcopy and cellblock techniques. For cervical cancer screening, p16 ${ }^{\mathrm{INK} 4 \mathrm{a}}$ is still an excellent surrogate marker useful for the detection of lesion likely to progress to malignancy. The cell cycle regulator $\mathrm{p} 16^{\text {INK4a }}$ is shown to be over-expressed in squamous dysplasia and cellblock together with colposcopy techniques can be used to improve further the "diagnostic accuracy of ASC-H Pap smears and to reduce unnecessary procedures" and overtreatment cases.

From the study, it can be concluded that cellblock with $\mathrm{H} \& \mathrm{E}$ staining and biomarkers $\mathrm{p} 16^{\mathrm{INK} 4 \mathrm{a}}$ is a useful tool for the confirmatory test of the cervical cytological specimen when examining premalignant lesions. The disparity arising from cellblock is due to deviation from the standardized procedures for cutting surfaces for excellent cellularity.

Through cellblock techniques, from this study proves to be a confirmatory test for the diagnosis of ASC-H Pap tests, "multiple unstained slides producing adequate cellularity can be obtained from each cell block" paving the way for additional markers that with increased the diagnostic specificity and sensitivity to be used and from this study, colposcopy technique. Cellblock techniques ties with colposcopy -the gold test of for cervical screening with some areas to be improved on the cell block.

Based on study findings, sensitivity ranges for colposcopy and cell block methods for cytodiagnosis, and in consideration of other aspects such as affordability of the services, loss of follow-ups, equipment required and capacity building, it can be concluded that cell block seems to be a better choice for the Kenyan population. With colposcopy, personnel needs to go through specialized training. Currently, the test is done by obstetrician/ gynecologists as compared to cellblock that can be done by a nurse or medical officer in the facility. Although the sensitivity, specificity and diagnostic accuracy of cell block vary, through this study, it is preferred as compared to colposcopy. Colposcopy faces challenges with efficiency, cost and comfort consideration. Most often, colposcopy should not be recommended to be done as a random biopsy for patients with normal-appearing cervices, meaning, primary test such as Pap smear must be done first and makes it more unaffordable and inaccessible to many patients. The sensitivity of colposcopy procedure can only be increased by taking two or more biopsies instead of one as the case of cell block where samples are obtained once.

\section{References}

1. D. M. Parkin, "Global cancer statistics in the year 2000," Lancet Oncology. 2001.

2. N. P. et al., "Efficacy of HPV DNA testing with cytology triage and/or repeat HPV DNA testing in primary cervical cancer screening," J. Natl. Cancer Inst., 2009.

3. L. Pantanowitz, M. Hornish, and R. Goulart, "The impact of digital imaging in the field of cytopathology," Cytojournal, 2009.

4. C. B. Visioli et al., "hr-HPV testing in the follow-up of women with cytological 
abnormalities and negative colposcopy," $\mathrm{Br}$. J. Cancer, 2013.

5. M. Safaeian, D. Solomon, and P. E. Castle, "Cervical Cancer Prevention-Cervical Screening: Science in Evolution," Obstetrics and Gynecology Clinics of North America. 2007.

6. P. Cristiani et al., "Rationale and development of an on-line quality assurance program for colposcopy in a population-based cervical screening setting in Italy," $B M C$ Health Serv. Res., 2013.

7. J. Li, M. J. Poi, and M. D. Tsai, "Regulatory mechanisms of tumor suppressor P16INK4A and their relevance to cancer," Biochemistry, 2011.

8. S. K. Ghosh et al., "Human papillomavirus testing for suspected cervical cancer patients from Southern Assam by fast-PCR.," Asian Pac. J. Cancer Prev., 2011.

9. V. B. Shidham, R. Mehrotra, G. Varsegi, K. D'Amore, B. Hunt, and R. Narayan, "P16 INK4a immunocytochemistry on cellblocks as an adjunct to cervical cytology: Potential reflex testing on specially prepared cellblocks from residual liquid-based cytology specimens," Cytojournal, 2011.

10. E. Burd, "Human papillomavirus and cervical cancerBurd E (2003) Human papillomavirus and cervical cancer. Clin Microbiol Rev 16: 1-17 Available at: http://www.sciencedirect.com/science/article/ pii/S0140673607614160," Clin Microbiol Rev, 2003.

11. A. Castanon, R. Landy, and P. D. Sasieni, "Is cervical screening preventing adenocarcinoma and adenosquamous carcinoma of the cervix," Int. J. Cancer, 2016.

12. J. L. Wang et al., "P16INK4a and laminin$5 \gamma 2$ chain expression during the progression of cervical neoplasia," Acta Oncol. (Madr)., 2006.

13. U. K.-E. et al., "Prediction of spontaneous regression of cervical intraepithelial neoplasia lesions grades 2 and 3 by proteomic analysis," Int. J. Proteomics, 2014.

14. S. Shrivastava, D. Barmon, P. Deka, and A. Kataki, "Vaginal vault carcinoma as second primary in a treated case of ovarian cancer," J. Midlife. Health, 2012.

15. S. Nishio et al., "P16INK4a immunohistochemistry is a promising biomarker to predict the outcome of low grade cervical intraepithelial neoplasia: Comparison study with HPV genotyping," $J$. Gynecol. Oncol., 2013.

16. R. Klaes et al., "p16INK4a immunohistochemistry improves interobserver agreement in the diagnosis of cervical intraepithelial neoplasia," Am. J. Surg. Pathol., 2002.

17. H. G. Kaspar and C. P. Crum, "The utility of immunohistochemistry in the differential diagnosis of gynecologic disorders," Arch. Pathol. Lab. Med., 2015.

18. D. Q. Liu et al., "Increased RIPK4 expression is associated with progression and poor prognosis in cervical squamous cell carcinoma patients," Sci. Rep., 2015.

19. F. S. Aslani, A. Safaei, M. Pourjabali, and M. Momtahan, "Evaluation of Ki67, p16 and CK17 markers in differentiating cervical intraepithelial Neoplasia and benign lesions," Iranian Journal of Medical Sciences. 2013.

20. E. R. Nijhuis et al., "An overview of innovative techniques to improve cervical cancer screening," Anal. Cell. Pathol., 2006.

21. I. Rufforny, E. J. Wilkinson, R. Redman, N. A. Massoll, and C. Liu, "p16ink4a Is Helpful in Discriminating Between Cervical Intraepithelial Neoplasia 1 and Equivocal Lesions of the Cervix," J. Low. Genit. Tract Dis., 2006.

22. I. Tsoumpou et al., "p16INK4 aimmunostaining in cytological and histological specimens from the uterine cervix: A systematic review and metaanalysis," Cancer Treatment Reviews. 2009. 